Toward Accurate Reaction Energetics for Molecular Line Growth at Surface: Quantum Monte Carlo and Density Functional Theory Calculations

Y. Kanai, N. Takeuchi

October 15, 2009

Journal of Chemical Physics 
This document was prepared as an account of work sponsored by an agency of the United States government. Neither the United States government nor Lawrence Livermore National Security, LLC, nor any of their employees makes any warranty, expressed or implied, or assumes any legal liability or responsibility for the accuracy, completeness, or usefulness of any information, apparatus, product, or process disclosed, or represents that its use would not infringe privately owned rights. Reference herein to any specific commercial product, process, or service by trade name, trademark, manufacturer, or otherwise does not necessarily constitute or imply its endorsement, recommendation, or favoring by the United States government or Lawrence Livermore National Security, LLC. The views and opinions of authors expressed herein do not necessarily state or reflect those of the United States government or Lawrence Livermore National Security, LLC, and shall not be used for advertising or product endorsement purposes. 


\title{
Toward Accurate Reaction Energetics for Molecular Line Growth at Surface: Quantum Monte Carlo and Density Functional Theory Calculations
}

\author{
Yosuke Kanai* \\ Berkeley Nanosciences and Nanoengineering Institute \\ University of California, Berkeley, CA, USA \\ Condensed Matter and Materials Division \\ Lawrence Livermore National Laboratory, Livermore, CA, USA
}

Noboru Takeuchi

Centro de Nanociencias y Nanotecnología, Universidad Nacional Autonoma de Mexico

Ensenada, Baja California, Mexico

* Corresponding author : kanai3@1lnl.gov

\begin{abstract}
We revisit the molecular line growth mechanism of styrene on the hydrogenated $\mathrm{Si}(001) 2 \times 1$ surface. In particular, we investigate the energetics of the radical chain reaction mechanism by means of diffusion quantum Monte Carlo (QMC) and density functional theory (DFT) calculations. For the exchange correlation (XC) functional we use the non-empirical generalized-gradient approximation (GGA) and meta-GGA. We find that the QMC result also predicts the intra dimer-row growth of the molecular line over the inter dimer-row growth, supporting the conclusion based on DFT results. However, the absolute magnitudes of the adsorption and reaction energies, and the heights of the energy barriers differ considerably between the QMC and DFT with the GGA/meta-GGA XC functionals.
\end{abstract}




\section{INTRODUCTION}

The organic functionalization of semiconductor surfaces is a promising route for the development of molecular nanostructures and organic-inorganic devices [1-6]. In particular, over the past several years, much attention has been focused on the formation of covalently attached nanostructures on silicon, starting from hydrogen-terminated $\mathrm{Si}(\mathrm{H}-\mathrm{Si})$ surfaces. On these surfaces, a radical chain reaction mechanism has been established to be operative when a Si dangling bond is initially present, created, for example, by the tip of a Scanning Tunneling Microscope (STM) or by ultraviolet (UV) light irradiation [7]. Several groups have extensively investigated fundamental aspects of the underlying mechanism and a qualitative understanding has been obtained through a number of theoretical works using density functional theory (DFT) calculations [8-11]. As the field matures, a more quantitative description of the reaction is desirable for a more rigorous comparison with experimental observations such as the growth rate of the molecular chains.

Predicting accurate transition rates from first-principles electronic structure calculations is a highly important challenge in general. There have been considerable efforts in advancing computational methodologies for efficiently locating the transition states and pathways in order to obtain the energy barriers. While a number of different methods have been developed [12] and the entropic effects can also be obtained accurately in many cases (e.g. via harmonic approximation or thermodynamic integration), an accurate computation of the potential energy barrier height remains as one of the greatest challenges. For molecular reactions, high-level quantum chemical methods such as coupled-cluster theory appear to provide reliable reaction energetics [13].

For surface reactions, however, we need to rely on alternative electronic structure methods that scale favorably with the number of electrons. For the present case of the surface radical reaction, it is quite important to validate DFT results, especially since they involve several challenging aspects for typical XC functionals; spinunpaired electrons, hydrogen atom transfers, and molecule-solid interactions. In this paper, we discuss the reaction energetics obtained using diffusion quantum Monte Carlo (QMC) calculations [14] and also DFT calculations with the non-empirical generalized gradient approximation (GGA) as well as the recent meta-GGA for the exchange correlation (XC) functional. 
In particular, we address the adsorption of styrene molecule and the subsequent hydrogen abstraction by the adsorbed molecule from a neighbor $\mathrm{SiH}$ group at surface, which comprise the principal step governing the competition between the inter dimer-row and intra dimer-row growths of the styrene molecular chain on the H$\operatorname{Si}(001) 2 x 1$ surface [11].

\section{METHOD}

Fixed-node diffusion QMC calculations are employed in order to obtain highly accurate total energies [15]. This method is based on solving the imaginary-time Schrödinger equation, $-\partial_{\tau} \Psi(R, \tau)=\left(\hat{H}-E_{0}\right) \Psi(R, \tau)$, which yields the many-body eigenstate with eigenvalue $\mathrm{E}_{0}$ as the imaginary-time $\tau$ goes to infinity. In practice, the integral form of this equation is employed with the short-time approximation to Green's function with a time step of 0.01 a.u using Monte Carlo (MC) method. Importance sampling is introduced with trial wavefunctions, which are obtained through a variance minimization [16] using variational MC from a Slater determinant of Kohn-Sham orbitals, multiplied by a two-body Jastrow factor. Troullier-Martin pseudopotentials (PPs) [17] are used for the core electrons together with localized basis sets of triple-zeta with triple-polarization quality for obtaining the Kohn-Sham orbitals [18]. Even with the fixed-node approximation employed here, the diffusion QMC typically gives an accuracy comparable to $\operatorname{CCSD}(\mathrm{T})$ as shown by Grossman in Ref. 19 for the G-1 molecule test set.

For DFT calculations [20], we employed both non-empirical GGA (PBE) [21] and meta-GGA (TPSS) [22] approximations to the XC functional in the psuedopotential-planewave formalism as implemented and tested

in Ref. 23. The meta-GGA depends on the kinetic energy density $\tau(r)=\frac{1}{2} \sum^{O c c .}\left|\nabla \phi_{i}^{K S}(r)\right|^{2}$ in addition to the electron density and its gradient as in the GGA approximation. PPs are generated according to Troullier-Martin procedures for PBE and TPSS XC approximations [17]. The kinetic energy cutoff for the planewave expansion of the wavefunction was 80 Rydberg. All results were obtained using the spin-unrestricted calculation. 
The string method implemented [24] in context of Car-Parrinello approach [25] was used to locate the minimum energy path (MEP) and the saddle point for obtaining accurate transition states (TS) with the atomic forces of less than $0.02 \mathrm{eV} / \mathrm{A}$ for all mobile atoms. The MEPs and the TSs were obtained at the level of DFT:GGA (PBE) since it was previously found that DFT:meta-GGA (TPSS) yielded essentially the same geometries, and differences in reaction energetics arise essentially from the $\mathrm{XC}$ approximation and not from the geometrical differences [23]. Geometry optimization at the level of diffusion QMC is at this time computationally too expensive for the surface reactions of interest even though there have been several recent advancements for computing forces in literature [26, 27]. We employed these computational approaches to obtain key reaction energetics for the principal step of the surface radical chain reaction mechanism, namely the hydrogen-atom abstraction process by the adsorbed Styrene molecule at the H-Si(001) 2x1 surface (Figure 1). In our periodic supercell approach, the surface is modeled by a slab of five (001) layers with twelve silicon atoms per layer and forming $(3 \times 4)$ surface cells in the $(001)$ plane. The molecules are adsorbed on the top surface of the slab, where $H$ atoms are present to saturate all $\mathrm{Si}$ dangling bonds $(\mathrm{db})$ but one, which corresponds to the reacting db that experimentally is created via the STM tip or UV radiation. At the bottom surface of the slab, all Si dbs are saturated by $\mathrm{H}$ atoms.

\section{RESULTS AND DISCUSSION}

The initial state of the reaction corresponds to the situation where the styrene molecule is far away from and is not interacting with the dangling bond of the $\mathrm{H}-\mathrm{Si}(001)$ surface. The adsorption energy at the intermediate state (Figure 1) is computed for both inter and intra dimer-row growths as shown in Table 1. QMC calculations predict a much larger adsorption energy than the DFT calculations. It is, however, quite surprising that the metaGGA does not appear to perform as well as the GGA with respect to the QMC. While the QMC calculation predicts the adsorption energy to be approximately $0.3 \sim 0.4 \mathrm{eV}$ larger than the GGA values, the meta-GGA values are $0.2 \sim 0.3 \mathrm{eV}$ smaller than the GGA values. It is important to note here that these are the total energy differences of two different configurations, as opposed to the total energies of the system, for which the meta-GGA consistently predicts values closer to that of QMC than the GGA as shown in Figure 3. 
Given this somewhat unexpected observation, we have also carried out all-electron calculations [28] with a large Gaussian basis set (aug-cc-pVQZ) on the related molecular reaction of styrene $+\mathrm{SiH} 3$. This is to make certain that our observation is not due to the use of the planewave-pseudopotential implementation [23]. The results are shown in Table 2, and the meta-GGA value deviates more than the GGA value from the QMC one. This is consistent with our observation in the surface reaction, and it indicates that the result is independent of the numerical implementation.

In the surface radical chain mechanism, the adsorbed styrene molecule abstracts a hydrogen atom from the neighboring Si-Si dimer of an adjacent dimer-row or the same dimer-row for the inter and intra dimer-row growth, respectively (Figure 1). As in the intermediate state, the same energetic trend was observed for the final state among all calculations (Table 1): the GGA appears to perform better than the meta-GGA for obtaining the reaction energies in this surface reaction. They both differ considerably from the QMC values.

The energy barrier is the crucial criterion for understanding the experimental observation of the intra dimer-row growth instead of the inter dimer-row growth for the formation of styrene molecular lines [9,10,11]. A number of theoretical works have addressed this issue using DFT calculations and they consistently have shown that the intra dimer-row growth is kinetically favored [29]. Our QMC results support this finding, predicting that the energy barrier for the intra dimer-row path is approximately $0.43 \mathrm{eV}$ smaller than the one for the inter dimerrow path. DFT results with the GGA and meta-GGA XC approximations yield energy barrier differences of 0.45 $\mathrm{eV}$ and $0.43 \mathrm{eV}$ for the two competing growth directions, respectively. Although the DFT calculations successfully discriminate between these two paths, the calculated DFT barrier heights are much smaller than the QMC values as shown in Table 1. It is quite interesting to note that with respect to the QMC results, the DFT:meta-GGA results are in better agreement than the DFT:GGA results for the energy barrier whereas the opposite trend was observed for calculating the stability of the intermediate and final states.

Deviations of the DFT total energies with respect to the QMC values (as seen in Figure 3) point to an interesting insight into the cause of the DFT energy barrier underestimations using the GGA and meta-GGA XC functionals. The total energy deviates less at the transition state than either at the intermediate or the final states, 
irrespective of the $\mathrm{XC}$ functional. This observation is consistent with the fact that most $\mathrm{XC}$ functionals are generally more accurate for electronic environments that are more homogeneous. Thus, for chemical reactions with a transition state of higher coordination, the DFT barrier height is generally underestimated as a result [30].

\section{CONCLUSION}

In conclusion, we have revisited the energetics of the surface radical chain reaction mechanism for a styrene molecular chain growth on the H-Si(001) 2x1 surface, using diffusion QMC and DFT calculations with GGA (PBE) and meta-GGA (TPSS) XC approximations. Somewhat unexpectedly, we found that the meta-GGA does not appear to perform as well as the GGA in obtaining adsorption and reaction energies. With respect to the QMC result, the reaction barrier is better described by the meta-GGA than the GGA. Importantly, we note here that meta-GGA consistently yields total energies that are in much better agreement with the QMC than the GGA (Figure 3). These observations reaffirm the importance of error cancellations in DFT for computing the reaction energetics. The QMC calculations support the DFT prediction of the lesser kinetic barrier for the intra dimer-row

over the inter dimer-row paths even though the absolute magnitudes of the DFT barriers are substantially underestimated, compared to the QMC values.

Although QMC calculations are computationally too demanding to be employed routinely, we have shown here that it is possible to apply such a high-accuracy calculation for assessing DFT results even for large systems like a surface because of the favorable scaling of the QMC computational cost with the number of electrons. Whereas high-accuracy quantum chemical calculations can be employed in cases of molecular reactions, for surface reactions QMC calculation is likely to be an important high-level method for assessing the DFT results, which need to be taken with caution for predicting some properties. With advancements in highperformance computational platforms, validating DFT results using QMC for surface reactions is likely to be more plausible in the future.

\section{ACKNOWLEDGEMNT}


YK would like to thank Jeffery C. Grossman for fruitful discussions on QMC methodologies. We thank DGSCA-UNAM supercomputing center for computational resources. Part of this work was performed under the auspices of the U.S. Department of Energy at Lawrence Livermore National Laboratory under Contract DE-AC52-07NA27344.

\section{REFERENCES}

[1] J. M. Buriak, Chemical Reviews, 102, 1271 (2002).

[2] S. F. Bent, Surf. Sci., 500, 879 (2002).

[3] R. A. Wolkow, Annu. Rev. Phys. Chem, 50, 413 (1999).

[4] F.A. Reboredo, E. Schwegier, G.J. Galli, Am. Chem. Soc., 125, 15243 (2003).

[5] P. L. Silvestrelli, O. Pulci, M. Palummo, R. Del Sole, and F. Ancilotto, Phys. Rev. B, 68, 235306 (2003).

[6] A. Calzolari, A. Ruini, E. Molinari, and J. M. Caldas, Phys. Rev. B, 73, 125420 (2006).

[7] G. P. Lopinsky, D. D. M. Wayner, R. A. Wolkow, Nature, 406, 48 (2000).

[8] N. Takeuchi, Y. Kanai, A. Selloni, J. Am. Chem. Soc., 126, 15890 (2004).

[9] J. H. Cho, D. H. Oh, L. Kleinman, Phys. Rev. B, 65, 081310 (R), (2002).

[10] X. Tong, G. A. DiLabio, R. A. Wolkow, Nano Lett, 4, 979 (2004).

[11] N. Takeuchi, and A. Selloni, J. Phys. Chem. B, 109, 11967 (2005).

[12] W. E, W Ren, and E. Vanden-Eijnden, Phys. Rev. B 66, 052301 (2002); H. Jonsson, G. Mills, and K. Jacobson, in Classical and Quantum Dynamics in Condensed Phase Simulations, edited by B. J. Berne, G. Ciccoti, and D. F. Coker, World Scientific, Singapore, 1998.; H. B. Schlegel. J. Comput. Chem. 3 214, (1982); G. T. Barkema, G. T. and N. Mousseau, Phys. Rev. Lett. 77, 4358 - 4361 (1996)

[13] For a recent review see, e.g., Rodney J. Bartlett and Monika Musial, Rev. Mod Phys., 79, 291 (2007).

[14] W. M. Foulkes, L. Mitas, R. J. Needs, and G. Rajagopal, Rev. Mod. Phys. 73, 33, (2001).

[15] QWalk code, L. K. Wagner, M. Bajdich, and L. Mitas, J. Comput. Phys. 228, 3390, (2009).

[16] C. Umrigar, K. Wilson, and J. Wilkins, Phys. Rev. Lett. 60, 1719 (1988). 
[17] N. Troullier, J. L. Martins, Phys. Rev., 43, 1993 (1991)

[18] SIESTA code, J. M. Soler, et al. J. Phys.: Condens. Matt. 14, 2745 (2002)

[19] J. C. Grossman, J. Chem. Phys. 117, 1434 (2002)

[20] QUANTUM-ESPRESSO code (www.quantum-espresso.org).

[21] J. P. Perdew, K. Burke, M. Ernzerholf, Phys. Rev. Lett., 77, 3865 (1996).

[22] J. Tao, J. P. Perdew, V. N. Staroverov, and G. E. Scuseria, Phys. Rev. Lett. 91, 146401 (2003)

[23] Y. Kanai, X. Wang, A. Selloni, R. Car, J. Chem. Phys., 125, 234104 (2006).

[24] Y. Kanai, A. Tilocca, A. Selloni, R. Car, J. Chem. Phys. 121, 3359 (2004).

[25] R. Car and M. Parinello, Phys. Rev. Lett. 55, 2471 (1985).

[26] S. Chiesa, D. M. Ceperley, and S. Zhang, Phys. Rev. Lett., 94, 036404 (2005).

[27] A. Badinski and R. J. Needs, Phys. Rev. B, 78, 035134 (2008).

[28] M. J. Frisch, G. W. Trucks, H. B. Schlegel et al., GAUSSIAN 03, Revision, C.02, Gaussian, Inc., Wallingford, CT, 2004.

[29] Quantitative values reported for the hydrogen abstraction barrier differ somewhat among them. This is likely due to minor but noticeable isomeric differences for the intermediate and transition states, N. Takeuchi, Y. Kanai, and A. Selloni, to be published.

[30] K. Burke, The ABC of DFT: http://dft.uci.edu/. 


\section{TABLES}

Table 1. Reaction energetics $(\mathrm{eV})$ of inter and intra dimer-row paths calculated using the DFT with GGA(PBE) and meta-GGA(TPSS) XC approximations, and the diffusion QMC. The energy reference $(E=0 \mathrm{eV})$ is the initial state, where Styrene and the $\mathrm{H}-\mathrm{Si}(001)$ surface are not interacting.

\begin{tabular}{|l|c|c|c|}
\hline Inter & GGA & meta-GGA & QMC \\
\hline Intermediate & -0.68 & -0.37 & $-1.0(1)$ \\
\hline Final & -1.02 & -0.72 & $-1.7(1)$ \\
\hline \hline Barrier & 1.09 & 1.20 & $1.4(1)$ \\
\hline
\end{tabular}

\begin{tabular}{|l|c|c|c|}
\hline Intra & GGA & meta-GGA & QMC \\
\hline \hline Intermediate & -0.65 & -0.41 & $-0.99(8)$ \\
\hline Final & -0.97 & -0.51 & $-1.7(1)$ \\
\hline \hline Barrier & 0.64 & 0.77 & $0.97(9)$ \\
\hline
\end{tabular}

Table 2. Comparison of All-electron (AE) and Pseudopotential (PP) calculations for computing "adsorption" energy $(\mathrm{eV})$ for Styrene $+\mathrm{SiH}_{3}$. AE calculations were performed with aug-cc-pVQZ Gaussian basis set. PP calculations were performed with the planewave expansion (80 Rydberg).

\begin{tabular}{|l|c|c|}
\hline & AE & PP \\
\hline \hline GGA & 1.21 & 1.20 \\
\hline meta-GGA & 1.13 & 1.10 \\
\hline \hline QMC & - & $1.29(2)$ \\
\hline
\end{tabular}




\section{FIGURES}

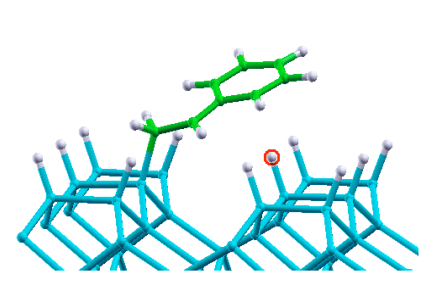

Intermediate

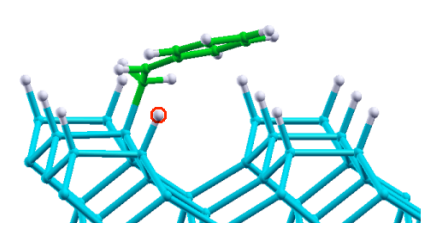

Intermediate

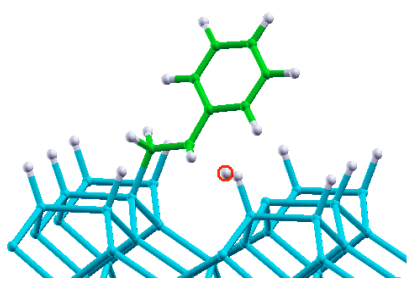

Transition State

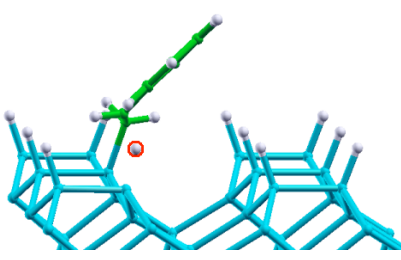

Transition State

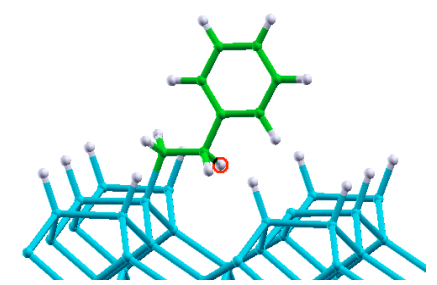

Final

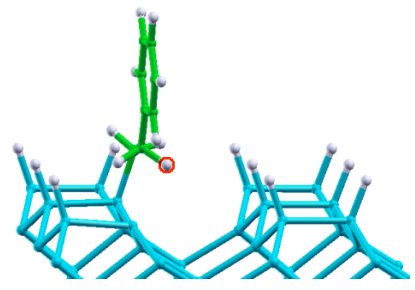

Final

Figure 1. Intermediate, Transition State, and Final states of the principal step for inter (TOP) and Intra (BOTTOM) dimer-row paths. The red circle indicates the surface hydrogen atom abstracted by the adsorbed Styrene molecule. See Ref. 11 for more details. 

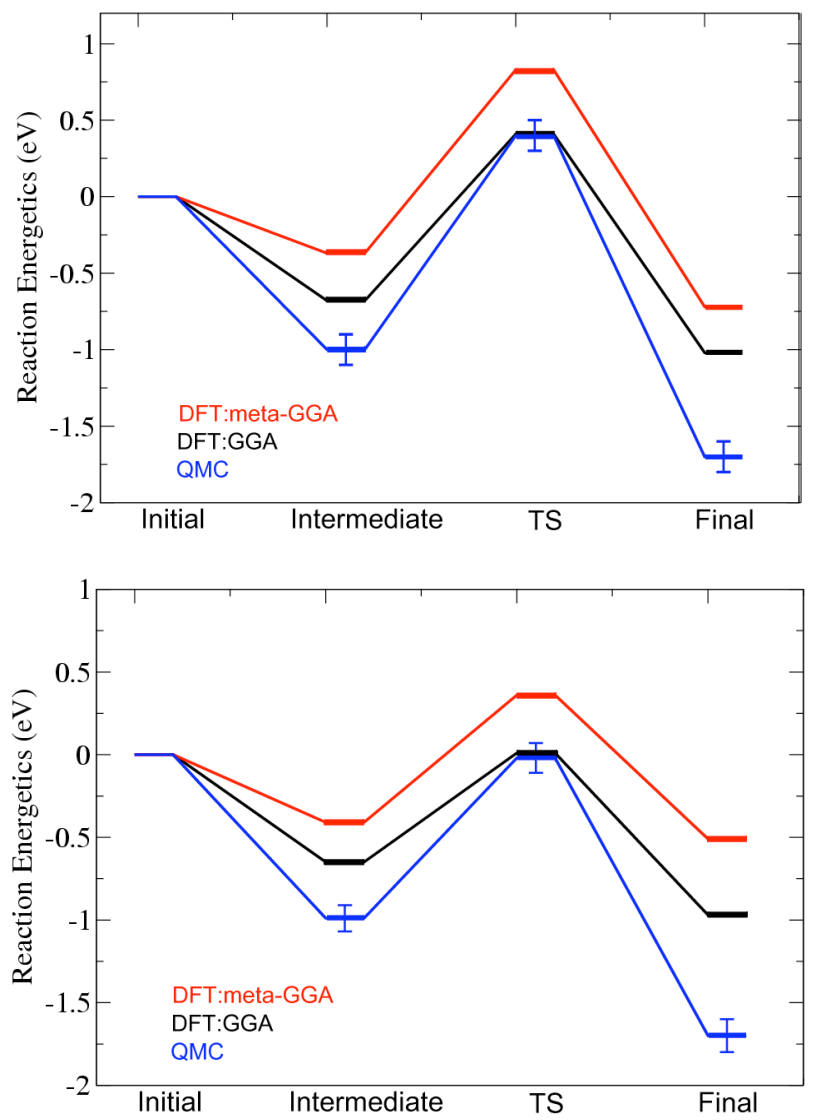

Figure 2. Reaction energetics $(\mathrm{eV})$ of intermediate, transition state (TS), and final states of the principal step for inter (TOP) and Intra (BOTTOM) dimer-row paths. The initial state is where Styrene and the H$\mathrm{Si}(001)$ surface are not interacting. 


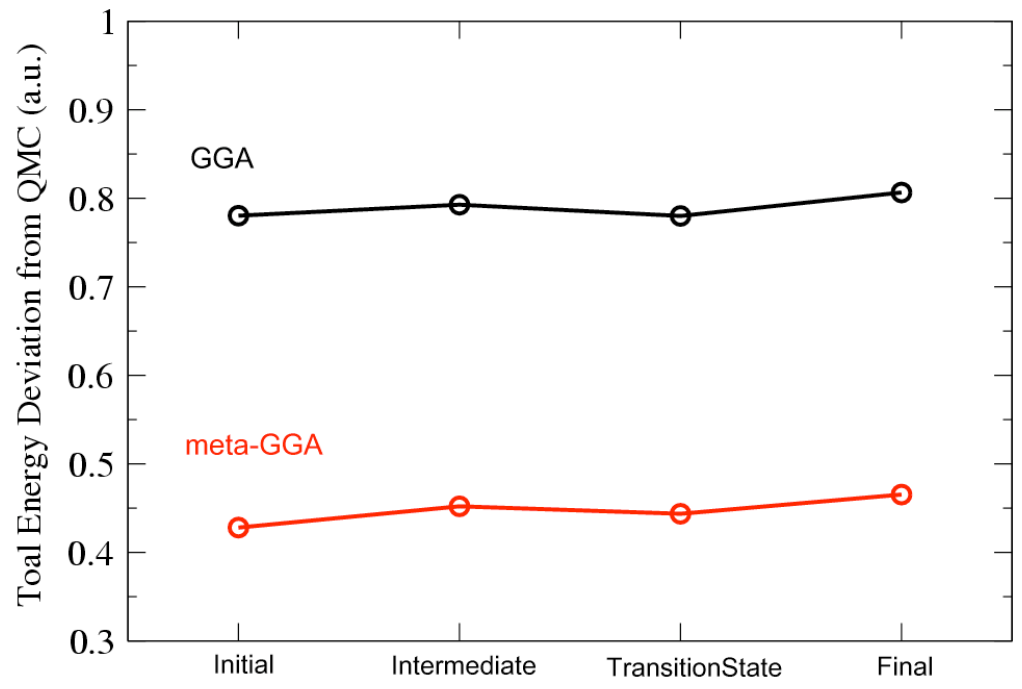

Figure 3. Deviation of the total energy (a.u.) from DFT calculations with the GGA (PBE) (black line) and the meta-GGA (TPSS) (red line) with respect to the diffusion QMC calculation for inter dimer-row path. The case for intra dimer-row path is indistinguishable at this scale and therefore not shown. 